\title{
Primary infective spondylodiscitis caused by Lactococcus garvieae and a review of human $L$. garvieae infections
}

\author{
J. F. W. Chan - P. C. Y. Woo · J. L. L. Teng • \\ S. K. P. Lau $\cdot$ S. S. M. Leung $\cdot$ F. C. C. Tam • \\ K.-Y. Yuen
}

Received: 12 October 2010/Accepted: 28 February 2011/Published online: 20 March 2011

(C) The Author(s) 2011. This article is published with open access at Springerlink.com

\begin{abstract}
We report the first case of primary infective spondylodiscitis due to Lactococcus garvieae, confirmed by $16 \mathrm{~S}$ rRNA gene sequencing, in the absence of concomitant endocarditis in a patient with long-standing gastritis on famotidine. He responded to a 6-week course of ampicillin. The gastrointestinal tract is probably the source of infection.
\end{abstract}

J. F. W. Chan · P. C. Y. Woo $(\bowtie) \cdot$ J. L. L. Teng ·

S. K. P. Lau - S. S. M. Leung - K.-Y. Yuen

Department of Microbiology, The University of Hong Kong, Queen Mary Hospital, 102 Pokfulam Road, Pokfulam,

Hong Kong Special Administrative Region, China

e-mail: pcywoo@hkucc.hku.hk

J. F. W. Chan

e-mail: jaspchan@gmail.com

P. C. Y. Woo · S. K. P. Lau · K.-Y. Yuen

State Key Laboratory of Emerging Infectious Diseases,

The University of Hong Kong, Queen Mary Hospital,

102 Pokfulam Road, Pokfulam, Hong Kong Special

Administrative Region, China

P. C. Y. Woo - S. K. P. Lau - K.-Y. Yuen

Research Centre of Infection and Immunology,

The University of Hong Kong, Queen Mary Hospital,

102 Pokfulam Road, Pokfulam, Hong Kong Special

Administrative Region, China

P. C. Y. Woo - S. K. P. Lau · K.-Y. Yuen

Carol Yu Centre for Infection, The University of Hong Kong, Queen Mary Hospital, 102 Pokfulam Road, Pokfulam,

Hong Kong Special Administrative Region, China

F. C. C. Tam

Department of Medicine, The University of Hong Kong,

Queen Mary Hospital, 102 Pokfulam Road, Pokfulam,

Hong Kong Special Administrative Region, China

\section{Introduction}

Lactococci are Gram-positive, catalase-negative, facultative anaerobic cocci in short chains or pairs traditionally considered to be of low virulence to human beings [1] Among the eight species and subspecies, Lactococcus garvieae is responsible for the majority of reported cases of opportunistic human infections in the literature. Similar to L. lactis [2], the most well-recognized clinical syndrome of human $L$. garvieae infections is infective endocarditis involving either native or prosthetic valves [3-8]. Other reported entities included liver abscess [9], peritonitis [7], diverticulitis [7], and infective spondylodiscitis in association with endocarditis [10]. Here, we describe a rare case of primary infective spondylodiscitis caused by $L$. garvieae in the absence of endocarditis in an immunocompetent patient who was successfully treated with antibiotics alone. After a thorough review of all of the sporadic reports in the literature, we conclude that the organism likely causes opportunistic infections in body systems with local defects by entering the bloodstream from diseased sites in the gastrointestinal tract after the ingestion of contaminated food.

\section{Case report}

A 70-year-old Chinese man with long-standing gastritis on histamine-2 receptor antagonist (famotidine $20 \mathrm{mg}$ twice daily) was admitted for fever and low back pain for 1 week. On admission, he had a temperature of $38.8^{\circ} \mathrm{C}$ and localized tenderness at the mid-lumbar spine. Blood tests showed a total white cell count of $8.67 \times 10^{9} 1^{-1}$ (normal, $4.40-10.10 \times 10^{9} 1^{-1}$ ) with $79.4 \%$ neutrophils (normal, $44.0-72.0 \%)$ and a neutrophil count of $6.89 \times 10^{9} 1^{-1}$ 
(normal, 2.2-6.7 $\times 10^{9} 1^{-1}$ ), raised C-reactive protein (CRP) and erythrocyte sedimentation rate (ESR) levels of $5.2 \mathrm{mg} \mathrm{dl}^{-1}$ (normal, $<0.76 \mathrm{mg} \mathrm{dl}^{-1}$ ) and $58 \mathrm{~mm} / \mathrm{h}$ (nor$\left.\mathrm{mal},<20 \mathrm{~mm} \mathrm{~h}^{-1}\right)$, respectively, and normal liver and renal function tests. A roentgenogram of the lumbar spine showed degenerative changes, including reduced lumbar lordosis and marginal osteophytes. Magnetic resonance imaging of the lumbar spine revealed abnormal contrast enhancement in the posterior aspect of the L3-4 disc and end-plate with surrounding soft tissue and epidural enhancement (Fig. 1). Culture of the percutaneous bone biopsy of L3-4 yielded tiny non-hemolytic colonies of Gram-positive cocci in short chains on 5\% sheep blood agar after $24 \mathrm{~h}$ of incubation in $5 \% \mathrm{CO}_{2}$ at $37^{\circ} \mathrm{C}$. The bacterium was grouped as Lancefield group $\mathrm{D}$, catalasenegative, bile esculin-positive, pyrrolidonyl arylamidasepositive, arginine-positive, vancomycin-susceptible, and did not grow in $6.5 \% \mathrm{NaCl}$. Although the Vitek (bioMérieux, Durham, NC, USA) system identified it as Enterococcus faecium with a $99 \%$ confidence level, the absence of growth in $6.5 \% \mathrm{NaCl}$ prompted the use of alternative identification methods. The API Rapid ID 32 Strep (bioMérieux, Marcy l'Etoile, France) and the BD

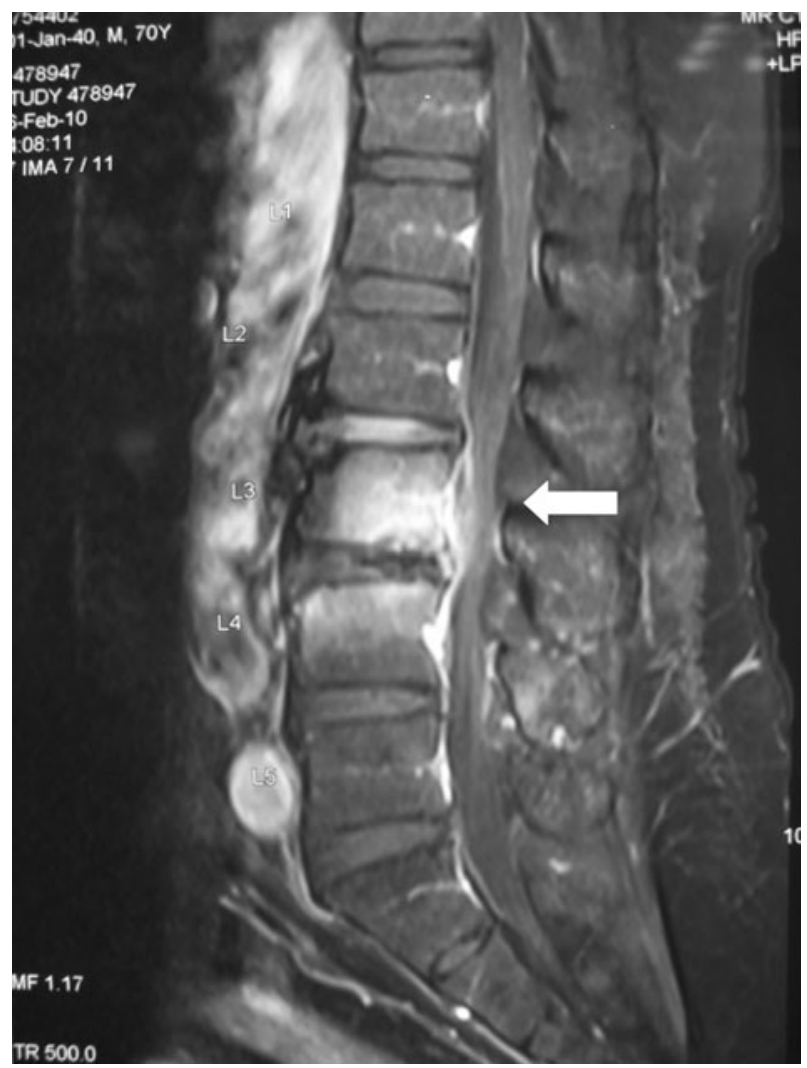

Fig. 1 T1-weighted magnetic resonance imaging of the lumbar spine showing abnormal contrast enhancement in the posterior aspect of the L3-4 disc and end-plate with surrounding soft tissue and epidural enhancement (arrow)
Phoenix $^{\mathrm{TM}}$ automated microbiology (Becton-Dickinson Diagnostics, Sparks, MD, USA) systems identified it as L. garvieae with 67.7 and $99 \%$ confidence levels, respectively.

16S rRNA gene sequencing of the Gram-positive coccus was performed according to our previous publications on the identification of other Gram-positive cocci [11, 12], using LPW8427 5'-GCGAACGGGTGAGTAACG-3' and LPW8428 5'-GACTTCACCCCAATCATC-3' (SigmaProligo, Singapore) as the polymerase chain reaction (PCR) and sequencing primers. The sequences of the PCR products were compared with the sequences of closely related species in GenBank by multiple sequence alignment using ClustalX 1.83 [13]. Phylogenetic relationships were determined using the neighbor-joining method. The results showed that there was no base difference between the 16S rRNA gene sequence of the Gram-positive coccus and that of $L$. garvieae (GenBank accession no. FJ915634.1), indicating unambiguously that the Grampositive coccus was a strain of L. garvieae (Fig. 2).

Based on the Clinical and Laboratory Standards Institute's (CLSI) recommendations on viridan streptococci, the organism was susceptible to ampicillin, vancomycin, ceftriaxone, intermediately resistant to penicillin with a minimum inhibition concentration of $1.0 \mu \mathrm{g} \mathrm{ml}^{-1}$, and resistant to clindamycin [14].

In view of previous reports on infective endocarditis associated with $L$. garvieae and a previous report of

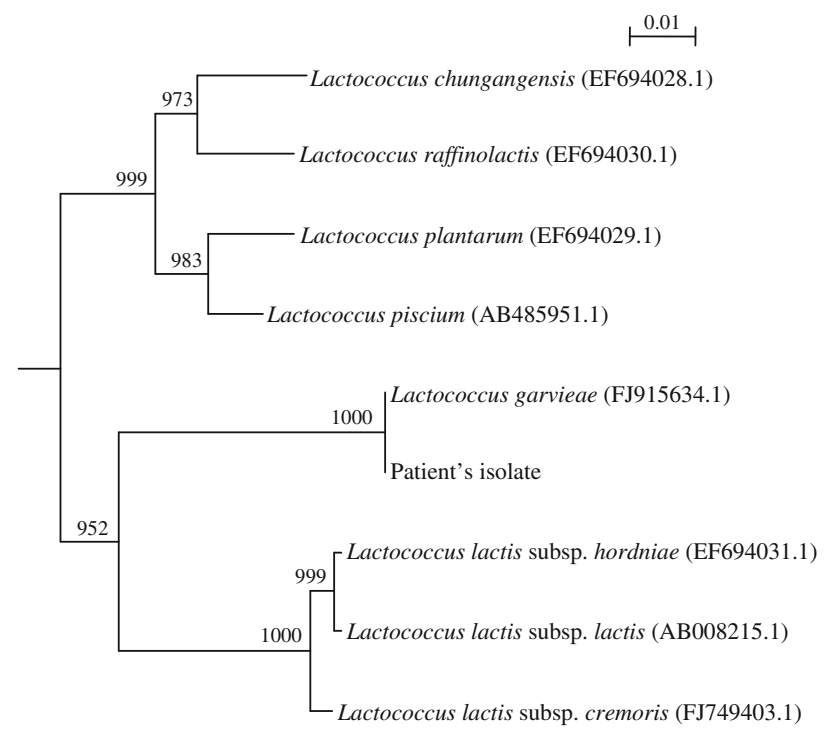

Fig. 2 Phylogenetic tree showing the relationships of the patient's isolate to other closely related Lactococcus species. The tree was inferred from $16 \mathrm{~S}$ rRNA data by the neighbor-joining method using Kimura's two-parameter correction and was rooted using the $16 \mathrm{~S}$ rRNA gene sequence of Streptococcus gallinaceus (NR_025453.1). Bootstrap values were calculated from 1,000 trees. The scale bar indicates the estimated number of substitutions per 100 bases. Names and accession numbers are given as cited in the GenBank database 
concomitant infective spondylitis and infective endocarditis [10], transthoracic echocardiogram was performed, which showed normal heart valves without any vegetation. Three sets of blood cultures taken before the use of antibiotics were negative and no clinically apparent immunologic or vasculitic phenomenon was observed. He was, thus, treated as a case of acute osteomyelitis with a 6-week course of intravenous ampicillin $2 \mathrm{~g}$ q6h and responded well clinically. The neutrophil count, CRP, and ESR levels normalized after 18 days of treatment. He was discharged 64 days after admission and remained well at follow-up 3 months afterwards.

\section{Discussion}

L. garvieae was separated from the Streptococcus genus in 1985 [15]. It was first isolated from bovines with mastitis [1] and, subsequently, from fish and human specimens [16]. It causes mastitis in ruminants, and lactococcosis, a septicemic infection characterized by hemorrhagic enteritis; bilateral exophthalmos; darkening of the skin; and congestion of the intestine, liver, kidney, spleen, and brain, in fish $[16,17]$. In the recent years, it has gained recognition as an emerging zoonotic pathogen, since an increasing number of cases of human infections due to L. garvieae have been encountered. Furutan et al. [18] first described the possible association between the organism and prosthetic valve endocarditis in 1991, although no clinical details were mentioned. Subsequently, including our patient, a total of 12 cases with clinical details have been reported in the English literature (Table 1). Six cases presented with infective endocarditis (five infecting native valves and one prosthetic valve) [3-8], one had liver abscess [9], one had septicemia with multiorgan failure [7], one had diverticulitis [7], one had secondary peritonitis [7], and one had infective spondylodiscitis associated with probable endocarditis [10]. Ten out of the 12 cases (83\%) had bacteremia. The present case is the first in describing L. garvieae infective spondylodiscitis in the absence of endocarditis and detectable bacteremia.

Although their clinical presentations vary, a number of similarities among these patients who developed infection due to this relatively avirulent organism can be observed by thorough analysis of the reported cases (Table 1). Concerning the probable pathogenesis, most of them started off with an anatomically or physiologically disturbed gastrointestinal tract $(9 / 12,75 \%)$, in the form of past surgery, active pathology, or the use of gastric acid suppressive therapy. As in our patient, who had regular visits to fish markets and intake of sashimi, the likely route of infection was often through the ingestion of contaminated fish [7]. Half of the cases had documented exposure to raw fish
$(6 / 12,50 \%)$, while the history was not mentioned in some of the other cases. The bacterium, after being acquired, then caused infection in the body systems with local predisposing factors $(9 / 12,75 \%)$, including valvular abnormalities in endocarditis, biliary tract tumor with prosthetic stenting in liver abscess, diverticular disease in septicemic diverticulitis, ruptured bowel in peritonitis, and degenerative spine in infective spondylodiscitis. These observations were well illustrated in our patient: chronic gastritis, longterm acid-suppressive therapy, regular intake of raw fish, and underlying degenerative spine. Thus, instead of developing infective spondylodiscitis due to other more common organisms $[19,20]$, our patient developed the disease secondary to $L$. garvieae.

Human infections caused by L. garvieae are associated with significant morbidity and mortality. For the nine patients who survived, most received prolonged courses (median 6 weeks) of antibiotics, mainly consisting of highdose $\beta$-lactams with or without aminoglycosides, and surgical interventions were also performed in three patients (Table 1). Of note, while clindamycin is often being used in cases of infective spondylodiscitis caused by other Gram-positive organisms such as staphylococci and streptococci, it would be ineffective against $L$. garvieae, as the organism is always resistant. Alternatively, oxacillin could be considered, as the bacterium is usually susceptible [21]. However, even despite appropriate antibiotic use and maximal supportive treatment, three patients $(3 / 12,25 \%)$ died as a result of cerebral hemorrhage complicating mitral valve replacement for infective endocarditis, hemobilia in cholangiocarcinoma with liver abscess, and multiorgan failure in concomitant Klebsiella pneumoniae and L. garvieae bacteremia (Table 1). This highlights the fact that, while three-quarters of cases of L. garvieae infections were treatable, a significant proportion could be fatal if serious complications, incurable comorbidities, or coexisting infections are present.

In summary, human infections due to $L$. garvieae are rare. The most common manifestation is infective endocarditis. Our case is the first case of primary infective spondylodiscitis in the absence of endocarditis. A thorough review of the literature suggests that the organism is probably acquired from the ingestion of contaminated food, especially raw fish, which then enters the bloodstream via anatomically or physiologically altered gastrointestinal tract, and, from there, spreads to infect distant sites with local predisposing pathologies. At-risk individuals, particularly those who have an anatomically or physiologically altered gastrointestinal tract, and coexisting local predisposing defects, especially valvular heart disease, are, therefore, discouraged from eating undercooked food, as this emerging zoonotic bacterium with supposedly low virulence is capable of causing serious infections with 


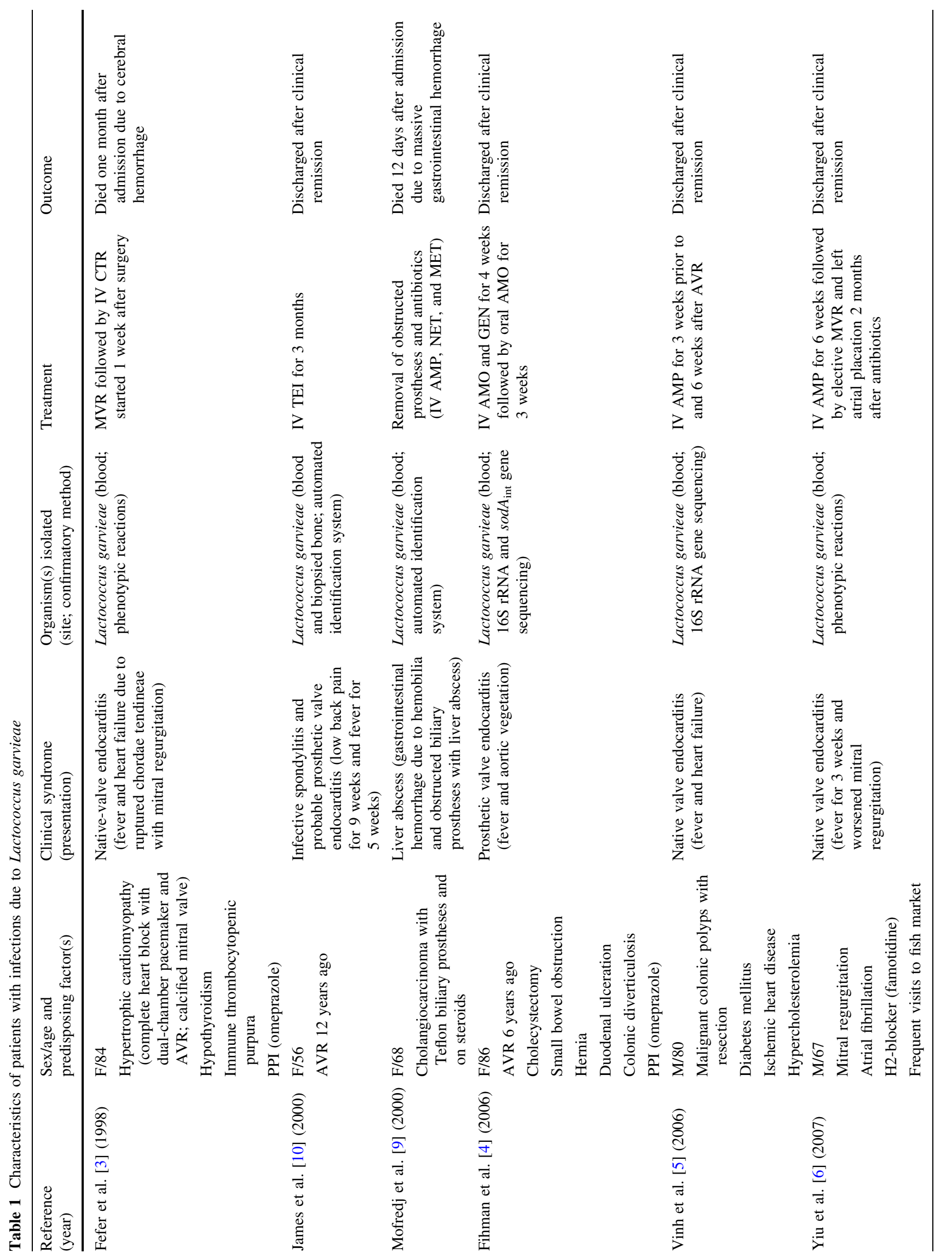




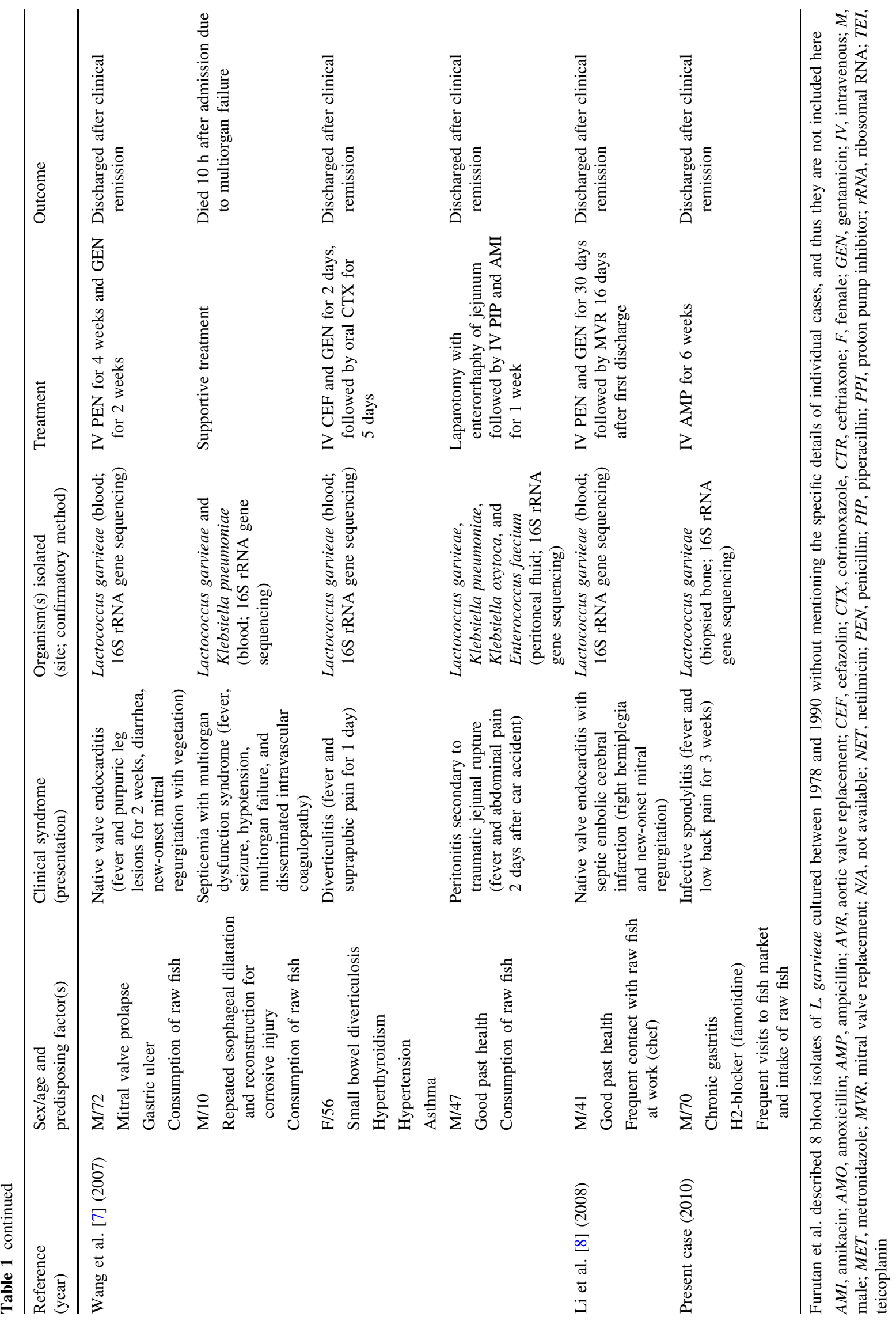


potential morbidities and mortalities. Accurate identification of the bacterium is essential and can be achieved by 16S rRNA gene sequencing.

Conflict of interest There is no conflict of interest.

Open Access This article is distributed under the terms of the Creative Commons Attribution Noncommercial License which permits any noncommercial use, distribution, and reproduction in any medium, provided the original author(s) and source are credited.

\section{References}

1. Collins MD, Farrow JA, Phillips BA, Kandler O. Streptococcus garvieae sp. nov. and Streptococcus plantarum sp. nov. J Gen Microbiol. 1983;129:3427-31.

2. Lin KH, Sy CL, Chen CS, Lee CH, Lin YT, Li JY. Infective endocarditis complicated by intracerebral hemorrhage due to Lactococcus lactis subsp. cremoris. Infection. 2010;38:147-9.

3. Fefer JJ, Ratzan KR, Sharp SE, Saiz E. Lactococcus garvieae endocarditis: report of a case and review of the literature. Diagn Microbiol Infect Dis. 1998;32:127-30.

4. Fihman V, Raskine L, Barrou Z, Kiffel C, Riahi J, Berçot B, et al. Lactococcus garvieae endocarditis: identification by $16 \mathrm{~S}$ rRNA and sodA sequence analysis. J Infect. 2006;52:e3-6.

5. Vinh DC, Nichol KA, Rand F, Embil JM. Native-valve bacterial endocarditis caused by Lactococcus garvieae. Diagn Microbiol Infect Dis. 2006;56:91-4.

6. Yiu KH, Siu CW, To KK, Jim MH, Lee KL, Lau CP, et al. A rare cause of infective endocarditis; Lactococcus garvieae. Int $\mathbf{J}$ Cardiol. 2007;114:286-7.

7. Wang CY, Shie HS, Chen SC, Huang JP, Hsieh IC, Wen MS, et al. Lactococcus garvieae infections in humans: possible association with aquaculture outbreaks. Int $\mathrm{J}$ Clin Pract. 2007;61:68-73.

8. Li WK, Chen YS, Wann SR, Liu YC, Tsai HC. Lactococcus garvieae endocarditis with initial presentation of acute cerebral infarction in a healthy immunocompetent man. Intern Med. 2008;47:1143-6.

9. Mofredj A, Baraka D, Kloeti G, Dumont JL. Lactococcus garvieae septicemia with liver abscess in an immunosuppressed patient. Am J Med. 2000;109:513-4.
10. James PR, Hardman SM, Patterson DL. Osteomyelitis and possible endocarditis secondary to Lactococcus garvieae: a first case report. Postgrad Med J. 2000;76:301-3.

11. Woo PC, Fung AM, Lau SK, Wong SS, Yuen KY. Group G betahemolytic streptococcal bacteremia characterized by $16 \mathrm{~S}$ ribosomal RNA gene sequencing. J Clin Microbiol. 2001;39: 3147-55.

12. Woo PC, Teng JL, Wu JK, Leung FP, Tse H, Fung AM, et al. Guidelines for interpretation of 16S rRNA gene sequence-based results for identification of medically important aerobic Grampositive bacteria. J Med Microbiol. 2009;58:1030-6.

13. Thompson JD, Gibson TJ, Plewniak F, Jeanmougin F, Higgins DG. The CLUSTAL_X windows interface: flexible strategies for multiple sequence alignment aided by quality analysis tools. Nucleic Acids Res. 1997;25:4876-82.

14. Clinical and Laboratory Standards Institute (CLSI). Performance standards for antimicrobial susceptibility testing; nineteenth informational supplement. CLSI Document M100-S19. 2009; 29(3). ISBN: 1-56238-690-5.

15. Schleifer KH, Kraus J, Dvorak C, Kilpper-Baelz R, Collins MD, Fischer W. Transfer of Streptococcus lactis and related streptococci to the genus Lactococcus gen. nov. Syst Appl Microbiol. 1985;6:183-95.

16. Vela AI, Vázquez J, Gibello A, Blanco MM, Moreno MA, Liébana $\mathrm{P}$, et al. Phenotypic and genetic characterization of Lactococcus garvieae isolated in Spain from lactococcosis outbreaks and comparison with isolates of other countries and sources. J Clin Microbiol. 2000;38:3791-5.

17. Vendrell D, Balcázar JL, Ruiz-Zarzuela I, de Blas I, Gironés O, Múzquiz JL. Lactococcus garvieae in fish: a review. Comp Immunol Microbiol Infect Dis. 2006;29:177-98.

18. Furutan NP, Breiman RF, Fischer MA, Facklam RR. Lactococcus garvieae infections in humans: a cause of prosthetic valve endocarditis (abstract C-297). Washington: 91st General Meeting American Society for Microbiology, 1991.

19. Honan M, White GW, Eisenberg GM. Spontaneous infectious discitis in adults. Am J Med. 1996;100:85-9.

20. Nigg AP, Schulze-Koops H, Wirth S, Weiss M, Bogner JR. Tuberculous spondylitis (Pott's disease). Infection. 2008;36:293-4.

21. Elliott JA, Facklam RR. Antimicrobial susceptibilities of Lactococcus lactis and Lactococcus garvieae and a proposed method to discriminate between them. J Clin Microbiol. 1996;34:1296-8. 